\title{
HIGHLIGHTS
}

\section{KIR status linked to susceptibility to ankylosing spondylitis}

KIR3DL1 and KIR3DS1, which are thought to be two alleles of a gene in the killer cell immunoglobulin-like receptor (KIR) family, are implicated in the development of ankylosing spondylitis (AS), report Zvyagin and colleagues in their study published in Cellular \& Molecular Immunology. "The observation that the inhibitory allele KIR3DL1 and the activating allele KIR3DS1 both contribute to the genetic risk of developing ankylosing spondylitis is the most significant finding of our work," says Dmitriy Chudakov, the study's corresponding author.

The KIR3DL1 and KIR3DS1 receptors are expressed on the surface of natural killer cells and a subpopulation of T lymphocytes, where they interact with HLA-B27, an MHC class I antigen strongly associated with AS and related diseases. The HLA-B27 gene is found in $>90 \%$ of patients with AS compared to $5-15 \%$ of individuals in the general population, but its presence accounts for only $16 \%$ of an individual's risk of developing the disease.
As KIR3DS1 occurs more frequently and KIR3DL1 less frequently in patients with AS than in healthy controls, the investigators expected that only the activating allele would influence disease risk. To test this hypothesis, they genotyped white Russian patients for functional $\left(K I R 3 D L 1^{\star} F\right)$ and nonfunctional (KIR3DL1 $\left.{ }^{\star} 004\right)$ alleles of the inhibitory receptor, as well as KIR3DS1. The study included 83 HLA-B27-positive patients with AS and 107 HLA-B27-positive healthy controls.

\section{1 ...the presence of KIR3DS1 has a stronger effect on disease risk than the absence of KIR3DL1*F 77}

Patients with AS had an increased frequency of the KIR3DS1 allele, independent of the presence or absence of $K I R 3 D L 1^{\star} F$. Thus, the presence of the activating allele seems to be crucial for disease initiation. By contrast, the homozygous KIR3DL1 ${ }^{\star} F$ genotype had a low frequency in patients with AS and a high frequency in healthy controls, despite a similar frequency of $K I R 3 D L 1^{*} 004$ in both groups. These findings indicate that the inhibitory allele actively protects against disease development, although the presence of KIR3DS1 has a stronger effect on disease risk than the absence of $K I R 3 D L 1^{\star} F$.

"Understanding that KIR3DL1 has a functional role in protection against ankylosing spondylitis is important for further deciphering the mechanisms of autoimmune diseases," concludes Chudakov. The researchers next plan to conduct functional studies of T lymphocytes carrying different KIR alleles and genome-wide association studies to identify other genes that contribute to the risk of AS.

Shreeya Nanda

Original article Zvyagin, I. V. et al. Contribution of functional KIR3DL1 to ankylosing spondylitis. Cell. Mol. Immunol. doi:10.1038/cmi.2010.42 